\title{
Rehabilitation of Salt-Affected Soil Through Residues Incorporation and Its Impact on Growth and Yield of Direct Seeded Rice and Wheat
}

ISSN: 2637-7659

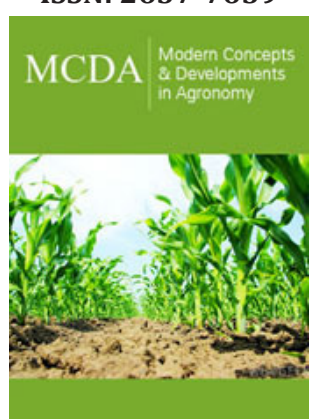

*Corresponding author: IA Mahmood, Land Resources Research Institute, Pakistan

Submission: 侮 March 22, 2019

Published: 制April 26, 2019

Volume 4 - Issue 1

How to cite this article: IA Mahmood, Hussain F, Shahzad A, Alam S, Ullah M, et al. Rehabilitation of Salt-Affected Soil Through Residues Incorporation and Its Impact on Growth and Yield of Direct Seeded Rice and Wheat. Mod Concep Dev Agrono.4(2). MCDA.000582.2019.

DOI: 10.31031/MCDA.2019.04.000582

Copyright@ IA Mahmood, This article is distributed under the terms of the Creative Commons Attribution 4.0 International License, which permits unrestricted use and redistribution provided that the original author and source are credited.

\author{
IA Mahmood ${ }^{1 *}$, Hussain $\mathrm{F}^{1}$, Shahzad $\mathrm{A}^{2}$, Alam SM${ }^{2}$, Ullah MA $^{1}$, Zaman ${ }^{1}$, Sultan \\ $\mathrm{T}^{1}$ and Hyder $\mathrm{SI}^{1}$
}

${ }^{1}$ Land Resources Research Institute, Pakistan

${ }^{2}$ National Institute for Genomics and Advanced Biotechnology, Pakistan

\begin{abstract}
A two year field study was conducted on a permanent layout to investigate the effect of crop residues (CR) incorporation and $\mathrm{P}$ application $\left(0,40,80,120 \mathrm{~kg} \mathrm{P}_{2} \mathrm{O}_{5} \mathrm{ha}^{-1}\right)$ on rehabilitation of saline soil $\left(\mathrm{EC}_{\mathrm{e}}=4.59\right.$ $\mathrm{dS} \mathrm{m}^{-1} ; \mathrm{pH}=8.38 ; \mathrm{CaCO}_{3}=3.21 \%$; Extractable $\mathrm{P}=4.07 \mathrm{mg} \mathrm{kg}^{-1}$; sandy clay loam) during 2011-12. The experiment was laid out according to split plot design with three replications. Planting of direct seeded rice (DSR) with and without crop residue incorporation @2ton ha ${ }^{-1}$ were placed in main plots and P application was in sub plots. Data on productive tillers, panicle length, paddy/grain and straw yields was collected. Soil was sampled $(0-15 \mathrm{~cm})$ before initiation and after the harvest of last crop. On an average of two years, maximum productive tillers (18), panicle length (33), paddy yield (3.26t ha- ${ }^{-1}$ ) and was produced with $\mathrm{P}$ application @ 80 $\mathrm{kg} \mathrm{P}_{2} \mathrm{O}_{5}$ ha $^{-1}$ along with CR incorporation. Similarly in case of wheat grown after DSR, maximum tillers (17), spike length (17), grain panicle ${ }^{-1}(66)$ and grain yield (3.56t ha-1) were produced with P application @80 $\mathrm{kg} \mathrm{P}_{2} \mathrm{O}_{5} \mathrm{ha}^{-1}$ along with CR incorporation. Although, the growth and yield contributing parameters with this treatment $\left(80 \mathrm{~kg} \mathrm{P}_{2} \mathrm{O}_{5}\right.$ ha $\left.^{-1}+\mathrm{CR}\right)$ performed statistically equal to $120 \mathrm{~kg} \mathrm{P}_{2} \mathrm{O}_{5}$ ha $^{-1}$ without $\mathrm{CR}$ incorporation during both the years, but on an average of two years, grain yield of DSR and wheat was significantly superior (22 and 24\% respectively) than that of higher P rate $\left(120 \mathrm{~kg} \mathrm{ha}^{-1}\right)$ without CR. Overall, continuous two year CR incorporation further increased (17\%) paddy yields during the follow up year of crop harvest. Higher concentration of $\mathrm{P}, \mathrm{K}$ and $\mathrm{Ca}^{2+}$ in both DSR and wheat plant tissues was found where $80 \mathrm{~kg} \mathrm{P}_{2} \mathrm{O}_{5}$ ha $^{-1}$ was applied along with CR incorporation or $120 \mathrm{~kg}$ $\mathrm{P}_{2} \mathrm{O}_{5}$ ha $^{-1}$ alone while $\mathrm{Na}^{+}$and $\mathrm{Mg}^{2+}$ concentration decreased with $\mathrm{CR}$ incorporation and increasing $\mathrm{P}$ rate. The soil salinity was decreased and fertility was improved significantly after two years of study.
\end{abstract}

Keywords: Slightly saline soil; Direct seeded rice; Crop residues incorporation; P application; Growth and yield; Ionic concentration; P use efficiency

Abbreviations: CR: Crop Residues; ECe: Electrical Conductivity of Extract: pH; Negative log of Hydrogen Ion; $\mathrm{CaCO}_{3}$ : Calcium Carbonate; P: Phosphorus; mg kg-1: milligram per kilogram; $\mathrm{kg}_{2} \mathrm{O}_{5}$ ha-1: Kilogram Phosphate per hectare; DSR: Direct Seeded Rice; $\mathrm{cm}$ : Centimeter; $\mathrm{t}$ ha ${ }^{-1}$ : ton per hectare; K: Potash; $\mathrm{N}^{\mathrm{at}}$ and $\mathrm{Mg}^{2+}$ : Sodium and Magnesium; PUE: Phosphorus Use Efficiency; SOP: Sulfate of Potash; SAR: Sodium Adsorption Ratio; SOM: Soil Organic Matter; TPU: Total P Uptake; LSD: Least Significant Difference; $\mathrm{CO}_{2}$ : Carbon di Oxide

\section{Introduction}

Soil salinity is a major problem in boosting up agricultural production throughout the world due to which million hectares of agricultural land is unable to produce potential crop yields. In regions such as Pakistan, over a quarter of the cultivatable land is occupied by medium to high salinity [1,2]. The problem is going faster because of heavy irrigations with brackish water which further decline the fertility of culturable lands and crop yields [26]. Saline soils contain surplus soluble salts $\left(\mathrm{Cl}^{-}\right.$and $\mathrm{SO}_{4}^{--}$of $\mathrm{Na}^{+}, \mathrm{Ca}^{2+}$ and $\left.\mathrm{Mg}^{2+}\right)$ which cause high osmotic pressure and compound interactions of $\mathrm{Na}$, $\mathrm{Ca}$ and $\mathrm{K}$ [7]. These salts disturb the equilibrium in rhizosphere and lessen crop productivity by accumulating hazardous ions. Adequate plant nutrition may reduce ill effects of these ions thereby helping plants to improve their growth under such situations [8-11]. Phosphorus availability in problem soils 
is affected by anion competition $\left(\mathrm{PO}_{4}^{--}\right.$and $\left.\mathrm{Cl}^{-}\right)$and many other interactions. Applied $\mathrm{P}$ is captured by $\mathrm{Ca}^{2+}$ present in calcareous soils and reacts with $\mathrm{P}$ to form insoluble dibasic $\mathrm{Ca}^{2+}$ phosphate compounds and is thus fixed. Much work has been reported regarding nutrient management for conventional crops to enhance their yields under unfavorable situation but much less exertion is reported on direct seeded rice under saline soils. There is a thirst to find out the economical application of nutrients especially of $\mathrm{P}$ and its availability to crop plants. Optimized P nutrition is critical for producing potential yields because it encourages healthy growth, development of strong root system, maximum tillering, uphold more flowering and seed formation [12].

Often, P deficiency in rice is referred to as "hidden hunger" which causes poor tillering, slow leaf canopy expansion, poor grain formation and delayed maturity. In our country, more than $90 \%$ soils are deficient in P [13]. There are so many factors responsible for low paddy yield production. Farming community in rice-wheat cropping system is facing water shortage, escalating fuel and high fertilizer cost (particularly of P fertilizers) and labour shortage during rice transplanting. About half of the rice cultivated area $(\sim$ $1.0 \mathrm{mha}$ ) is salt-affected which has moderate to high salinity, high $\mathrm{pH}$ and shortage of good quality ground water causing 30-70\% paddy yield reduction [14]. Moreover, $\mathrm{P}$ availability in soluble orthophosphate form is a prevalent limitation under calcareous soils because it makes insoluble compounds and does not release plant available $\mathrm{P}$ even upon heavy irrigations. Although, a large proportion of applied $\mathrm{P}$ in the soil becomes immobile due to this process $[11,15]$. However, the plants readily utilize only $8-33 \%$ of applied P in the first growing season [16]. Hence, there is a need to increase the use of $\mathrm{P}$ fertilizers in order to guarantee food security for ever increasing populations. Soils containing insufficient amounts of plant-available $\mathrm{P}$ not only produce economically deplorable yields, but other inputs are also used less efficiently. Thus, there is an urgent need to seek out strategies by which $\mathrm{P}$ fertilizers can be used more effectively in such farming systems where $P$ is deficient and where its use is economically practicable. Most of the farmers of rice-wheat cropping system burn wheat crop residues to prepare their lands for timely rice transplanting. This practice not only despoil the environment but also the precious source of plant nutrients, main source of organic material and the important constituent for soil health are being smashed on large scale in this agricultural ecosystem. About $25 \%$ of $\mathrm{N}$ and $\mathrm{P}$, $50 \%$ of the $\mathrm{S}$, and $75 \%$ of $\mathrm{K}$ uptake by cereal crops are retained in crop residues, making them valuable nutrient sources. Since large portion of plant nutrients taken up by plants remains in the straw, much of this can be recycled for subsequent crop growth after its decomposition $[11,17]$. In many studies, recycling of crop residues is reported to increase the organic carbon, nutrient contents, increased crop yields [18-20].

Direct seeding of rice is a new and the most suitable technology for resource poor community of salt-affected lands for which transplanting labour cost, water and machinery tools expenses required during puddling and transplanting could be saved in addition to timely sowing of rice. The crop matures early as compared to traditional transplanting which further makes possible sowing of wheat crop well in time after the harvest of rice. Besides these benefits, there is much more plant population under direct seeded rice as compared to traditionally transplanted rice for which nutrients requirement could also be higher to produce potential yields. A little work is reported regarding nutrient management particularly of $\mathrm{P}$ application for direct seeded rice in salt-affected soils of Pakistan. Therefore, keeping all these factors in mind, a long-term field study was planned to investigate appropriate $\mathrm{P}$ dose for direct seeded rice along with wheat straw incorporating and their impact on paddy yield in a slightly saline soil of district Hafizabad.

\section{Materials and Methods}

A two year study under a permanent layout was conducted in marginal saline soil of rice-wheat cropping system at farmers field in district Hafizabad of Pakistan having $\mathrm{EC}_{\mathrm{e}}=4.59 \mathrm{dS} \mathrm{m} \mathrm{m}^{-1}, \mathrm{pH}=8.38$, $\mathrm{SAR}=6.57\left(\mathrm{mmol}_{\mathrm{c}} \mathrm{L}^{-1}\right)^{1 / 2}$, Extractable $\mathrm{P}=4.07 \mathrm{mgkg}^{-1}, \mathrm{CaCO}_{3}=3.21 \%$, and sandy clay loam texture during 2011 and 2012 (Table 1-5). The experiment was laid out according to split plot design with three replications. Planting methods i.e., direct seeding with and without crop residue (wheat) incorporation @2 t ha ${ }^{-1}$ were kept in main plots and various $\mathrm{P}$ doses (0, 40, 80 and $120 \mathrm{~kg} \mathrm{P}_{2} \mathrm{O}_{5}$ ha $\left.^{-1}\right)$ were applied in sub plots. Recommended basal dose of $\mathrm{N} @ 100 \mathrm{~kg}$ $\mathrm{ha}^{-1}$ (half at sowing time and remaining half at tillering stage) and $\mathrm{K}$ $@ 50 \mathrm{~kg} \mathrm{ha}^{-1}$ as SOP were applied to all the plots at the time of sowing. Soaked seed (for 24) of rice cv. Supper-2000 @40kg ha-1 was broadcasted uniformly. The same inputs were applied to intermediate wheat crop. Effective weedicides were used to control weeds and the crop was grown to maturity. All agronomic requirements and plant protection measures were met throughout the growth period whenever required. Pre-sowing and after the harvest final crop soil samples $(0-15 \mathrm{~cm}$ depth) were collected for ECe, SAR, $\mathrm{pH}$, Soil Organic Matter (SOM), P, K Ca contents analysis (Table 6 \& 7) according to the methods suggested by Ryan et al. [21]. Plant samples were collected at maturity for the determination of ionic concentration in tissues. Dried and ground samples were digested in perchloric-nitric acid (2:1 $1 \mathrm{~N})$ mixture [22] to estimate $\mathrm{P}, \mathrm{K}, \mathrm{Na}^{+}$, $\mathrm{Ca}^{2+}$ and $\mathrm{Mg}^{2+}$ concentration in plant tissues by spectronic-20 and atomic absorption spectrophotometer. At maturity, the crop was harvested and agronomic data on fertile tillers, plant height, panicle length, grains panicle ${ }^{-1}, 1000$-grain weight, paddy and straw yields were recorded. Phosphorus use efficiency (PUE) was computed by using the following formula as suggested by Fageria et al. [23]

$$
P U E(\%)=\frac{\left(T P U_{F}\right)-\left(T P U_{C}\right)}{\text { Pdose }\left(k g h a^{-1}\right)} \times 100
$$

Where $\mathrm{TPU}_{\mathrm{F}}$ is Total P uptake (kg ha $\mathrm{k}^{-1}$ ) in Fertilized Plots and $\mathrm{TPU}_{\mathrm{C}}$ is Total P uptake $\left(\mathrm{kg} \mathrm{ha}^{-1}\right)$ in Control Plots. Total PU was calculated as:

$$
\operatorname{TPU}\left(\mathrm{kg} \mathrm{ha}^{-1}\right)=\frac{P \text { contents }(\%) \text { in plant party }(\text { dry matter }) \times \text { Yield }\left(\mathrm{kg} \mathrm{ha}^{-1}\right)}{100}
$$


Table 1: Growth and yield of direct seeded rice influenced by $\mathrm{P}$ application and CR incorporation under saline soil (Average of three repeats).

\begin{tabular}{|c|c|c|c|c|c|c|c|c|c|c|c|c|}
\hline \multirow{3}{*}{$\begin{array}{c}P_{2} \mathbf{O}_{5} \\
(\mathbf{k g} \\
\left.\mathrm{ha}^{-1}\right)\end{array}$} & \multicolumn{2}{|c|}{ Tillers } & \multicolumn{2}{|c|}{ Plant Height (cm) } & \multicolumn{2}{|c|}{$\begin{array}{l}\text { Panicle Length } \\
\text { (cm) }\end{array}$} & \multicolumn{2}{|c|}{ Grain Panicle ${ }^{-1}$} & \multicolumn{2}{|c|}{$\begin{array}{c}\text { Paddy Yield } \\
\text { ha }^{-1} \text { ) }\end{array}$} & \multicolumn{2}{|c|}{$\begin{array}{r}\text { Straw Yield } \\
\text { ha' }^{-1} \text { ) }\end{array}$} \\
\hline & $+\mathrm{CR}$ & $-\mathbf{C R}$ & $+\mathrm{CR}$ & $-\mathrm{CR}$ & $+\mathrm{CR}$ & $-\mathrm{CR}$ & $+\mathrm{CR}$ & $-\mathbf{C R}$ & $+\mathbf{C R}$ & - CR & $+\mathrm{CR}$ & $-\mathbf{C R}$ \\
\hline & \multicolumn{12}{|c|}{2011} \\
\hline 0 & $13.67 \mathrm{bc}$ & $8.00 \mathrm{~d}$ & $126.33 \mathrm{a}$ & $118.00 \mathrm{~b}$ & $26.00 \mathrm{bc}$ & $17.67 \mathrm{~d}$ & $108.67 \mathrm{bc}$ & $78.33 \mathrm{~d}$ & $2.497 \mathrm{~cd}$ & $1.887 \mathrm{e}$ & $\begin{array}{c}6.430 \\
\mathrm{ab}\end{array}$ & $4.760 \mathrm{c}$ \\
\hline 40 & $14.00 \mathrm{bc}$ & $12.33 \mathrm{c}$ & $129.00 \mathrm{a}$ & $119.67 \mathrm{~b}$ & $27.00 \mathrm{bc}$ & $22.00 \mathrm{c}$ & $110.67 \mathrm{~b}$ & $99.33 \mathrm{c}$ & $2.637 \mathrm{bc}$ & $2.215 \mathrm{~d}$ & $6.590 \mathrm{a}$ & $5.657 \mathrm{~b}$ \\
\hline 80 & $17.67 \mathrm{a}$ & $\begin{array}{c}15.67 \\
a b\end{array}$ & $130.00 \mathrm{a}$ & $120.67 \mathrm{~b}$ & $30.67 \mathrm{a}$ & $24.67 \mathrm{bc}$ & 119.33 a & $105.67 \mathrm{bc}$ & $2.987 \mathrm{a}$ & $2.707 \mathrm{abc}$ & $6.980 \mathrm{a}$ & $6.523 \mathrm{ab}$ \\
\hline 120 & $16.00 \mathrm{ab}$ & $\begin{array}{c}16.00 \\
\mathrm{ab}\end{array}$ & $130.00 \mathrm{a}$ & $120.67 \mathrm{~b}$ & $28.00 \mathrm{ab}$ & $27.00 \mathrm{ab}$ & $115.67 \mathrm{a}$ & $114.33 \mathrm{ab}$ & $\begin{array}{c}2.852 \\
\mathrm{ab}\end{array}$ & $2.817 \mathrm{abc}$ & $6.830 \mathrm{a}$ & $6.737 \mathrm{a}$ \\
\hline \multirow[t]{2}{*}{ LSD } & \multicolumn{2}{|c|}{2.8362} & \multicolumn{2}{|c|}{4.6787} & \multicolumn{2}{|c|}{3.1727} & \multicolumn{2}{|c|}{8.36} & \multicolumn{2}{|c|}{0.2639} & \multicolumn{2}{|c|}{0.9532} \\
\hline & \multicolumn{12}{|c|}{2012} \\
\hline 0 & $14.67 \mathrm{bc}$ & $9.33 \mathrm{~d}$ & $125.67 \mathrm{~b}$ & $98.00 \mathrm{~d}$ & $29.33 \mathrm{bc}$ & $17.00 \mathrm{e}$ & $105.67 \mathrm{de}$ & $86.00 \mathrm{f}$ & $2.873 \mathrm{bc}$ & $1.560 \mathrm{e}$ & $7.223 \mathrm{~b}$ & $5.090 \mathrm{~d}$ \\
\hline 40 & $15.00 \mathrm{bc}$ & $13.00 \mathrm{c}$ & $\begin{array}{c}127.00 \\
\mathrm{ab}\end{array}$ & $114.00 \mathrm{c}$ & $31.00 \mathrm{~b}$ & $24.00 \mathrm{~d}$ & $113.33 \mathrm{bc}$ & $99.00 \mathrm{e}$ & $\begin{array}{l}3.103 \\
\text { abc }\end{array}$ & $2.127 \mathrm{~d}$ & $7.313 \mathrm{~b}$ & $5.750 \mathrm{c}$ \\
\hline 80 & $18.67 \mathrm{a}$ & $\begin{array}{c}15.67 \\
a b\end{array}$ & $133.67 \mathrm{a}$ & $115.33 \mathrm{c}$ & $35.67 \mathrm{a}$ & $25.33 \mathrm{~cd}$ & $122.67 \mathrm{a}$ & $109.00 \mathrm{~cd}$ & $3.537 \mathrm{a}$ & $2.723 c$ & $7.913 \mathrm{a}$ & $6.542 \mathrm{~b}$ \\
\hline 120 & $16.33 \mathrm{ab}$ & $\begin{array}{c}16.33 \\
\mathrm{ab}\end{array}$ & $\begin{array}{l}129.00 \\
\mathrm{ab}\end{array}$ & $116.33 \mathrm{c}$ & $31.67 \mathrm{~b}$ & $\begin{array}{c}26.67 \\
\text { bcd }\end{array}$ & $118.67 \mathrm{ab}$ & $115.00 \mathrm{ab}$ & $\begin{array}{l}3.308 \\
a b\end{array}$ & $3.307 \mathrm{ab}$ & $\begin{array}{l}7.510 \\
\mathrm{ab}\end{array}$ & $6.730 \mathrm{~b}$ \\
\hline LSD & \multicolumn{2}{|c|}{2.4983} & \multicolumn{2}{|c|}{7.794} & \multicolumn{2}{|c|}{3.7621} & \multicolumn{2}{|c|}{5.6257} & \multicolumn{2}{|c|}{0.5246} & \multicolumn{2}{|c|}{0.5817} \\
\hline
\end{tabular}

Means followed by same letter(s) do not differ significantly at $\mathrm{P} \leq 0.05 \mathrm{NS}=$ Non-significant.

Table 2: Growth and yield of wheat influenced by P application and CR incorporation under saline soil (Average of three repeats).

\begin{tabular}{|c|c|c|c|c|c|c|c|c|c|c|c|c|}
\hline \multirow{2}{*}{$\begin{array}{c}P_{2} \mathbf{O}_{5}(\mathrm{~kg} \\
\left.\mathrm{ha}^{-1}\right)\end{array}$} & \multicolumn{2}{|c|}{ Tillers } & \multicolumn{2}{|c|}{ Plant Height (cm) } & \multicolumn{2}{|c|}{$\begin{array}{l}\text { Spike Length }{ }^{-1} \\
\text { (cm) }\end{array}$} & \multicolumn{2}{|c|}{ Grain Spike $^{-1}$} & \multicolumn{2}{|c|}{ Grain Yield $\left(\mathrm{t} \mathrm{ha}^{-1}\right)$} & \multicolumn{2}{|c|}{ Straw Yield $\left(\mathrm{t} \mathrm{ha}^{-1}\right)$} \\
\hline & $+\mathrm{CR}$ & $-\mathrm{CR}$ & $+\mathrm{CR}$ & $-\mathrm{CR}$ & $+\mathbf{C R}$ & - CR & $+\mathrm{CR}$ & $-\mathrm{CR}$ & $+\mathrm{CR}$ & $-\mathrm{CR}$ & $+\mathrm{CR}$ & $-\mathrm{CR}$ \\
\hline 0 & $9.33 \mathrm{~d}$ & 6.67 e & $81.00 \mathrm{ab}$ & $71.00 \mathrm{~b}$ & $\begin{array}{c}12.67 \\
\text { cd }\end{array}$ & $9.67 \mathrm{e}$ & $33.00 \mathrm{c}$ & $20.67 d$ & $2.652 \mathrm{c}$ & $1.591 \mathrm{e}$ & $5.936 \mathrm{ab}$ & $3.476 \mathrm{~d}$ \\
\hline 40 & $12.33 \mathrm{c}$ & $8.67 \mathrm{de}$ & 88.33 a & $74.33 \mathrm{~b}$ & $\begin{array}{c}14.33 \\
\text { bc }\end{array}$ & $11.00 \mathrm{de}$ & $46.67 \mathrm{~b}$ & $42.67 \mathrm{~b}$ & $3.194 \mathrm{abc}$ & $2.054 \mathrm{~d}$ & $6.314 \mathrm{ab}$ & $4.433 \mathrm{c}$ \\
\hline 80 & $17.00 \mathrm{a}$ & $14.00 \mathrm{bc}$ & 89.67 a & $78.67 \mathrm{ab}$ & $17.33 \mathrm{a}$ & $12.67 \mathrm{~cd}$ & 56.33 a & $43.67 \mathrm{~b}$ & $3.560 \mathrm{a}$ & $2.877 \mathrm{bc}$ & $6.676 \mathrm{a}$ & $5.447 \mathrm{~b}$ \\
\hline 120 & $14.33 \mathrm{bc}$ & $16.33 \mathrm{ab}$ & 89.33 a & $79.00 \mathrm{ab}$ & $\begin{array}{c}15.33 \\
\mathrm{ab}\end{array}$ & $16.33 \mathrm{ab}$ & $48.33 \mathrm{~b}$ & $46.00 \mathrm{~b}$ & $3.259 \mathrm{ab}$ & $3.320 \mathrm{ab}$ & $6.377 \mathrm{ab}$ & $6.547 \mathrm{a}$ \\
\hline LSD & \multicolumn{2}{|c|}{2.4807} & \multicolumn{2}{|c|}{9.2534} & \multicolumn{2}{|c|}{2.5848} & \multicolumn{2}{|c|}{6.2967} & \multicolumn{2}{|c|}{0.42} & \multicolumn{2}{|c|}{0.9954} \\
\hline
\end{tabular}

Means followed by same letter(s) do not differ significantly at $\mathrm{P} \leq 0.05 \mathrm{NS}=$ Non-significant.

Table 3: Ionic concentration (\%) in DSR straw affected by P application and CR incorporation under saline soil (Average of three repeats).

\begin{tabular}{|c|c|c|c|c|c|c|c|c|}
\hline \multirow{3}{*}{$\begin{array}{c}P_{2} \mathbf{O}_{5}(\mathrm{~kg} \\
\left.\mathrm{ha}^{-1}\right)\end{array}$} & \multicolumn{2}{|c|}{$\mathbf{K}$} & \multicolumn{2}{|c|}{$\mathrm{Na}$} & \multicolumn{2}{|c|}{$\mathrm{Ca}$} & \multicolumn{2}{|c|}{ Mg } \\
\hline & $+\mathrm{CR}$ & - CR & $+\mathrm{CR}$ & - CR & $+\mathrm{CR}$ & - CR & $+\mathrm{CR}$ & - CR \\
\hline & \multicolumn{8}{|c|}{2011} \\
\hline 0 & $1.906 \mathrm{e}$ & $1.827 \mathrm{e}$ & $0.276 \mathrm{bc}$ & $0.459 \mathrm{a}$ & $0.196 \mathrm{c}$ & $0.091 \mathrm{~d}$ & $0.196 \mathrm{~b}$ & $0.389 \mathrm{a}$ \\
\hline 40 & $2.268 \mathrm{de}$ & $2.167 \mathrm{de}$ & $0.266 \mathrm{bc}$ & $0.315 \mathrm{~b}$ & $0.234 \mathrm{c}$ & $0.212 \mathrm{c}$ & $0.195 \mathrm{~b}$ & $0.374 \mathrm{a}$ \\
\hline 80 & $3.445 \mathrm{a}$ & $2.555 \mathrm{~cd}$ & $0.144 \mathrm{e}$ & $0.230 \mathrm{~cd}$ & $0.322 \mathrm{a}$ & $0.247 \mathrm{bc}$ & $0.141 \mathrm{~b}$ & $0.390 \mathrm{a}$ \\
\hline 120 & $2.920 \mathrm{~b}$ & $2.992 \mathrm{~b}$ & $0.178 \mathrm{de}$ & 0.209 cde & $0.298 \mathrm{ab}$ & $0.320 \mathrm{a}$ & $0.135 \mathrm{~b}$ & $0.355 \mathrm{a}$ \\
\hline LSD & \multicolumn{2}{|c|}{0.4237} & \multicolumn{2}{|c|}{0.0473} & \multicolumn{2}{|c|}{0.0618} & \multicolumn{2}{|c|}{0.0738} \\
\hline
\end{tabular}




\begin{tabular}{|c|c|c|c|c|c|c|c|c|}
\hline & \multicolumn{8}{|c|}{2012} \\
\hline 0 & $2.016 \mathrm{de}$ & $1.666 \mathrm{e}$ & $0.225 \mathrm{bc}$ & $0.419 \mathrm{a}$ & $0.126 \mathrm{~d}$ & $0.085 \mathrm{e}$ & $0.120 \mathrm{c}$ & $0.215 \mathrm{a}$ \\
\hline 40 & $2.748 \mathrm{bc}$ & $2.044 \mathrm{de}$ & $0.178 \mathrm{~cd}$ & $0.290 \mathrm{ab}$ & $0.197 \mathrm{c}$ & $0.116 \mathrm{de}$ & $0.115 \mathrm{~cd}$ & $0.174 \mathrm{~b}$ \\
\hline 80 & $3.645 \mathrm{a}$ & $2.289 \mathrm{~cd}$ & $0.138 \mathrm{~d}$ & $0.228 \mathrm{bc}$ & $0.291 \mathrm{a}$ & $0.196 \mathrm{c}$ & $0.094 \mathrm{~d}$ & $0.143 \mathrm{c}$ \\
\hline 120 & $3.053 \mathrm{~b}$ & $2.739 \mathrm{bc}$ & $0.184 \mathrm{~cd}$ & $0.199 \mathrm{~cd}$ & $0.249 \mathrm{~b}$ & $0.276 \mathrm{ab}$ & $0.107 \mathrm{~d}$ & $0.106 \mathrm{~d}$ \\
\hline LSD & \multicolumn{2}{|c|}{0.4977} & \multicolumn{2}{|c|}{0.0734} & \multicolumn{2}{|c|}{0.0331} & \multicolumn{2}{|c|}{0.0275} \\
\hline
\end{tabular}

Means followed by same letter(s) do not differ significantly at $\mathrm{P} \leq 0.05 \mathrm{NS}=$ Non-significant

Table 4: Ionic concentration (\%) in DSR paddy influenced by $\mathrm{P}$ application and crop residues (CR) incorporation under saline soil (Average of three repeats)

\begin{tabular}{|c|c|c|c|c|c|c|c|c|}
\hline \multirow{3}{*}{$\begin{array}{c}P_{2} \mathbf{O}_{5}(\mathrm{~kg} \\
\left.\text { ha }^{-1}\right)\end{array}$} & \multicolumn{2}{|c|}{ K } & \multicolumn{2}{|c|}{$\mathrm{Na}$} & \multicolumn{2}{|c|}{$\mathrm{Ca}$} & \multicolumn{2}{|c|}{ Mg } \\
\hline & $+\mathrm{CR}$ & - CR & $+\mathrm{CR}$ & $-\mathrm{CR}$ & $+\mathrm{CR}$ & $-\mathrm{CR}$ & $+\mathrm{CR}$ & $-\mathrm{CR}$ \\
\hline & \multicolumn{8}{|c|}{2011} \\
\hline 0 & $0.790 \mathrm{f}$ & $0.264 \mathrm{~g}$ & $0.127 \mathrm{bc}$ & $0.241 \mathrm{a}$ & 0.142 c & $0.077 \mathrm{~d}$ & $0.134 \mathrm{~b}$ & $0.196 \mathrm{a}$ \\
\hline 40 & $1.310 \mathrm{de}$ & $1.032 \mathrm{ef}$ & $0.116 \mathrm{bc}$ & $0.171 \mathrm{~b}$ & $0.194 \mathrm{~b}$ & $0.156 \mathrm{bc}$ & $0.106 \mathrm{~cd}$ & $0.189 \mathrm{a}$ \\
\hline 80 & $1.853 \mathrm{a}$ & $1.385 \mathrm{~cd}$ & $0.087 \mathrm{c}$ & $0.137 \mathrm{bc}$ & $0.298 \mathrm{a}$ & $0.188 \mathrm{bc}$ & 0.079 e & $0.183 \mathrm{a}$ \\
\hline 120 & $1.570 \mathrm{bc}$ & $1.658 \mathrm{ab}$ & $0.098 \mathrm{c}$ & $0.104 \mathrm{c}$ & $0.272 \mathrm{a}$ & $0.251 \mathrm{a}$ & $0.098 \mathrm{de}$ & $0.123 \mathrm{bc}$ \\
\hline \multirow[t]{2}{*}{ LSD } & \multicolumn{2}{|c|}{0.2035} & \multicolumn{2}{|c|}{0.0473} & \multicolumn{2}{|c|}{0.0327} & \multicolumn{2}{|c|}{0.0213} \\
\hline & \multicolumn{8}{|c|}{2012} \\
\hline 0 & $0.835 \mathrm{~d}$ & $0.282 \mathrm{e}$ & $0.149 \mathrm{bc}$ & $0.212 \mathrm{a}$ & $0.165 \mathrm{~cd}$ & $0.045 \mathrm{e}$ & $0.112 \mathrm{~cd}$ & $0.235 \mathrm{a}$ \\
\hline 40 & $1.325 \mathrm{bc}$ & $1.136 \mathrm{c}$ & $0.096 \mathrm{~d}$ & $0.159 \mathrm{~b}$ & $0.226 \mathrm{~b}$ & $0.154 \mathrm{~d}$ & 0.099 cde & $0.177 \mathrm{~b}$ \\
\hline 80 & $1.854 \mathrm{a}$ & $1.391 \mathrm{~b}$ & $0.063 \mathrm{e}$ & $0.117 \mathrm{~cd}$ & $0.291 \mathrm{a}$ & $0.192 \mathrm{bc}$ & $0.044 \mathrm{e}$ & $0.129 \mathrm{bc}$ \\
\hline 120 & $1.674 \mathrm{a}$ & $1.759 \mathrm{a}$ & $0.085 \mathrm{de}$ & $0.089 \mathrm{de}$ & $0.265 \mathrm{a}$ & $0.219 \mathrm{~b}$ & $0.068 \mathrm{de}$ & $0.063 \mathrm{de}$ \\
\hline LSD & \multicolumn{2}{|c|}{0.1875} & \multicolumn{2}{|c|}{0.032} & \multicolumn{2}{|c|}{0.0358} & \multicolumn{2}{|c|}{0.0567} \\
\hline
\end{tabular}

Means followed by same letter(s) do not differ significantly at $\mathrm{P} \leq 0.05 \mathrm{NS}=$ Non-significant

Table 5: Ionic concentration (\%) in wheat plant tissues influenced by $\mathrm{P}$ application and crop residues (CR) incorporation under saline soil (Average of three repeats).

\begin{tabular}{|c|c|c|c|c|c|c|c|c|}
\hline \multirow{3}{*}{$\begin{array}{c}\mathbf{P}_{2} \mathbf{O}_{5}(\mathrm{~kg} \\
\left.\mathrm{ha}^{-1}\right)\end{array}$} & \multicolumn{2}{|c|}{$\mathbf{K}$} & \multicolumn{2}{|c|}{$\mathrm{Na}$} & \multicolumn{2}{|c|}{$\mathrm{Ca}$} & \multicolumn{2}{|c|}{ Mg } \\
\hline & $+\mathrm{CR}$ & - CR & $+\mathrm{CR}$ & - CR & $+\mathrm{CR}$ & $-\mathrm{CR}$ & $+\mathrm{CR}$ & - CR \\
\hline & \multicolumn{8}{|c|}{ Straw } \\
\hline 0 & $1.249 \mathrm{c}$ & $1.163 \mathrm{c}$ & 0.136 bc & $0.221 \mathrm{a}$ & $0.124 \mathrm{~d}$ & $0.105 \mathrm{~d}$ & $0.143 \mathrm{bc}$ & $0.216 \mathrm{a}$ \\
\hline 40 & $1.896 \mathrm{ab}$ & $1.148 \mathrm{c}$ & $0.104 \mathrm{de}$ & 0.207 a & $0.211 \mathrm{~b}$ & $0.177 \mathrm{c}$ & $0.135 \mathrm{bcd}$ & $0.198 \mathrm{a}$ \\
\hline 80 & $2.100 \mathrm{a}$ & $1.800 \mathrm{~b}$ & 0.089 e & $0.169 \mathrm{~b}$ & $0.293 \mathrm{a}$ & $0.205 \mathrm{bc}$ & $0.106 \mathrm{~cd}$ & $0.149 \mathrm{~b}$ \\
\hline 120 & $1.923 \mathrm{ab}$ & $1.874 \mathrm{ab}$ & $0.097 \mathrm{e}$ & $0.135 \mathrm{~cd}$ & $0.219 \mathrm{~b}$ & $0.222 \mathrm{~b}$ & $0.121 \mathrm{bcd}$ & $0.102 \mathrm{~d}$ \\
\hline \multirow[t]{2}{*}{ LSD } & \multicolumn{2}{|c|}{0.2321} & \multicolumn{2}{|c|}{0.031} & \multicolumn{2}{|c|}{0.0329} & \multicolumn{2}{|c|}{0.0403} \\
\hline & \multicolumn{8}{|c|}{ Grain } \\
\hline 0 & $0.992 \mathrm{~d}$ & $0.532 \mathrm{e}$ & $0.192 \mathrm{ab}$ & $0.207 \mathrm{a}$ & $0.124 \mathrm{bc}$ & $0.059 \mathrm{~d}$ & $0.177 \mathrm{a}$ & $0.194 \mathrm{a}$ \\
\hline 40 & $1.180 \mathrm{~cd}$ & $0.994 \mathrm{~d}$ & $0.144 \mathrm{c}$ & $0.174 \mathrm{~b}$ & $0.173 \mathrm{~b}$ & $0.108 \mathrm{c}$ & $0.118 \mathrm{bc}$ & $0.168 \mathrm{a}$ \\
\hline 80 & $1.738 \mathrm{a}$ & $1.356 \mathrm{bc}$ & $0.098 \mathrm{f}$ & $0.138 \mathrm{~cd}$ & $0.253 \mathrm{a}$ & $0.139 \mathrm{bc}$ & $0.076 \mathrm{~d}$ & $0.137 \mathrm{~b}$ \\
\hline 120 & $1.517 \mathrm{ab}$ & $1.575 \mathrm{ab}$ & $0.116 \mathrm{de}$ & 0.110 ef & $0.180 \mathrm{~b}$ & $0.181 \mathrm{~b}$ & $0.094 \mathrm{~cd}$ & $0.106 \mathrm{~cd}$ \\
\hline LSD & \multicolumn{2}{|c|}{0.2484} & \multicolumn{2}{|c|}{0.017} & \multicolumn{2}{|c|}{0.0472} & \multicolumn{2}{|c|}{0.0271} \\
\hline
\end{tabular}

Means followed by same letter(s) do not differ significantly at $\mathrm{P} \leq 0.05 \mathrm{NS}=$ Non-significant. 
The data thus, collected were subjected to statistical analysis using software package MSTAT-C and treatment means were compared using least significant difference (LSD) at $5 \%$ probability level [24].

\section{Results}

\section{Growth and yield of DSR}

On an average of two years data, maximum fertile tillers (19), panicle length $(34 \mathrm{~cm})$, grain panicle ${ }^{-1}(121)$ and paddy yield (3.26t ha $\mathrm{h}^{-1}$ ) were produced with P application @ 80kg $\mathrm{P}_{2} \mathrm{O}_{5} \mathrm{ha}^{-1}$ along with $\mathrm{CR}$ incorporation during first and second cropping years, respectively (Table 2). Although, these parameters were statistically at par with P application @ $120 \mathrm{~kg} \mathrm{P}_{2} \mathrm{O}_{5}$ ha $^{-1}$ without CR incorporation during both the years but grain yield harvested with $\mathrm{P}$ application @ 80 $\mathrm{kg}_{2} \mathrm{O}_{5}$ ha $^{-1}$ along with CR incorporation was significantly superior when compared with higher $\mathrm{P}$ rate $(120 \mathrm{~kg}$ ha $^{-1}$ ) without CR during both the cropping years. On an average of two-year data, paddy produced by this treatment showed $22 \%$ additional yield over control ( $\left.0 \mathrm{~kg} \mathrm{P} \mathrm{ha}^{-1}+\mathrm{CR}\right)$. Under CR incorporation, further increase in $\mathrm{P}$ application $\left(120 \mathrm{~kg} \mathrm{P}_{2} \mathrm{O}_{5} \mathrm{ha}^{-1}\right)$ caused 6\% paddy yield reduction as compared to the $\mathrm{P}$ application @ 80kg $\mathrm{P}_{2} \mathrm{O}_{5} \mathrm{ha}^{-1}$. During second cropping year, interestingly the lower dose of $\mathrm{P}\left(40 \mathrm{~kg} \mathrm{P}_{2} \mathrm{O}_{5} \mathrm{ha}^{-1}+\mathrm{CR}\right)$ performed reasonably better but was slightly comparable with higher rate of $\mathrm{P}\left(120 \mathrm{~kg} \mathrm{P}_{2} \mathrm{O}_{5}\right.$ ha $^{-1}$ ) without CR incorporation. Overall, continuous two-year CR incorporation further increased $17 \%$ paddy yield during the follow up year of crop harvest as compared to previous crop harvest under CR incorporation.

\section{Growth and yield of wheat crop}

Although, under CR incorporation, minimum number of productive tillers (9.33), spike length $(12.67 \mathrm{~cm})$, grain spike ${ }^{-1}$ (33.00) and grain yield (2.87t ha $\left.\mathrm{h}^{-1}\right)$ were produced from the plots where no $\mathrm{P}$ was applied but these parameter were significantly higher than that of control treatment $\left(0 \mathrm{~kg} \mathrm{P}_{2} \mathrm{O}_{5}\right.$ ha $\left.^{-1}\right)$ without $\mathrm{CR}$ incorporation (Table 3). A considerable increase in growth and yield components, straw and grain yields of wheat grown were observed with $\mathrm{P}$ fertilization and $\mathrm{CR}$ incorporation. Maximum fertile tillers (17), spike length $(17 \mathrm{~cm})$, grain panicle ${ }^{-1}(56)$ and grain yield (3.56t ha $\mathrm{t}^{-1}$ ) was produced with P application @ 80kg $\mathrm{P}_{2} \mathrm{O}_{5}$ ha $^{-1}$ along with CR incorporation, which was obviously superior (7\%) than the higher $\mathrm{P}$ application $\left(120 \mathrm{~kg} \mathrm{P}_{2} \mathrm{O}_{5}\right.$ ha $\left.^{-1}\right)$ rate without CR incorporation. After the harvest of first DSR crop, the wheat grain yield harvested with $\mathrm{P}$ application under CR incorporation even in lesser amount $\left(40 \mathrm{~kg} \mathrm{P}_{2} \mathrm{O}_{5}\right.$ ha $\left.^{-1}\right)$ performed slightly equal to higher $\mathrm{P}$ rate $\left(120 \mathrm{~kg} \mathrm{P}_{2} \mathrm{O}_{5}\right.$ ha $\left.^{-1}\right)$ without CR incorporation. When $\mathrm{P}$ was applied without $\mathrm{CR}$ incorporation, maximum grain yield was obtained with increasing the rate of $\mathrm{P}$ and was the highest where $\mathrm{P}$ was applied @ $120 \mathrm{~kg} \mathrm{P}_{2} \mathrm{O}_{5}$ ha $^{-1}$ but was not as much as harvested with $80 \mathrm{~kg} \mathrm{P}_{2} \mathrm{O}_{5}$ ha $^{-1}$ under CR incorporation.

\section{Ionic concentration and PUE of DSR and Wheat}

It is evident from the data in Table $4 \& 5$ that high $\mathrm{Na}^{+}$and $\mathrm{Mg}^{2+}$ while low $\mathrm{K}^{+}$and $\mathrm{Ca}^{2+}$ concentrations were determined from both DSR and wheat plant tissues where no $\mathrm{P}$ was applied without CR incorporation. Phosphorus application and CR incorporation significantly reduced saline ions $\left(\mathrm{Na}^{+}\right.$and $\left.\mathrm{Mg}^{2+}\right)$ concentration and improved $\mathrm{K}^{+}$and $\mathrm{Ca}^{2+}$ in both DSR and wheat crop plants. The data indicated that $\mathrm{P}$ application particularly with $\mathrm{CR}$ incorporation considerably decreased $\mathrm{Na}^{+}$concentration and increased $\mathrm{K}^{+}$and $\mathrm{Ca}^{2+}$ concentration in plant tissues. Phosphorus application still at lower rate $\left(40 \mathrm{~kg} \mathrm{P}_{2} \mathrm{O}_{5} \mathrm{ha}^{-1}\right)$ along with CR incorporation performed better or even statistically equal to higher dose of $\mathrm{P}\left(120 \mathrm{~kg} \mathrm{P}_{2} \mathrm{O}_{5}\right.$ ha $^{-1}$ ) without CR incorporation. Similarly, maximum PUE in case of DSR (26\%) and wheat (23\%) was reflected from the plots treated with $80 \mathrm{~kg} \mathrm{P}_{2} \mathrm{O}_{5}$ ha $^{-1}$ application under CR incorporation (Figure 1) which was comparable with higher $\mathrm{P}$ dose $\left(120 \mathrm{~kg} \mathrm{P}_{2} \mathrm{O}_{5}\right.$ ha $\left.^{-1}\right)$ under no CR incorporation. Under CR incorporation, further increase in $\mathrm{P}$ application (120 $\mathrm{kg} \mathrm{P}_{2} \mathrm{O}_{5} \mathrm{ha}^{-1}$ ) did not showed significant difference in PUE by both the crops. On the other hand, when P was applied without CR incorporation, maximum PUE was determined from the treatments where $\mathrm{P}$ was applied at higher rate $\left(120 \mathrm{~kg} \mathrm{P}_{2} \mathrm{O}_{5} \mathrm{ha}^{-1}\right)$ and was not as much of $\mathrm{P}$ application @80 $\mathrm{kg} \mathrm{P}_{2} \mathrm{O}_{5}$ ha $^{-1}$ under CR incorporation.

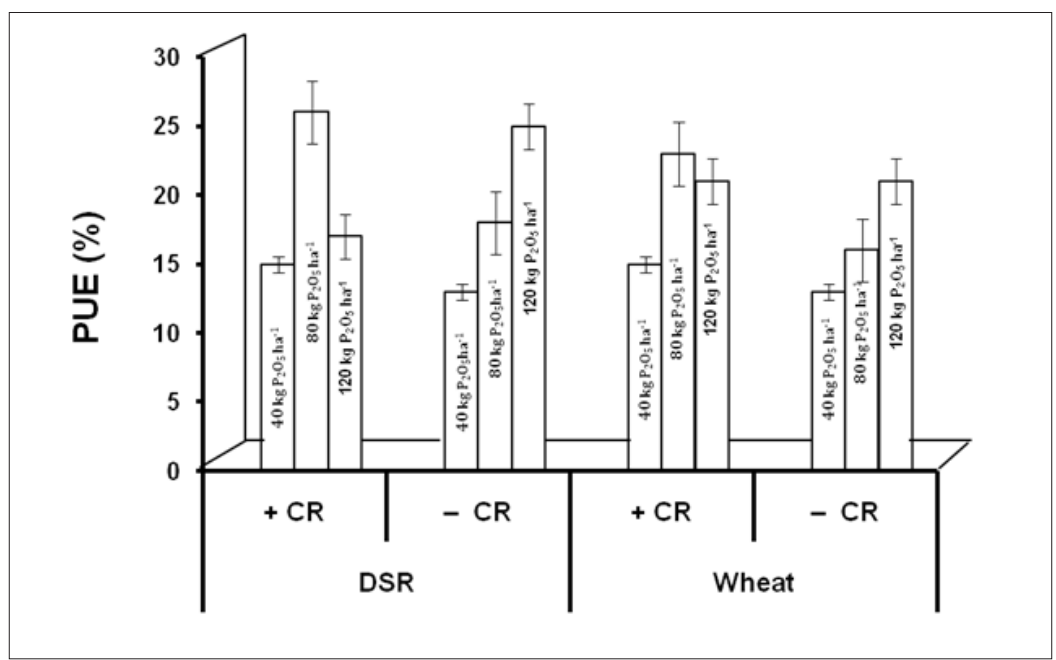

Figure 1: PUE (\%) of DSR and Wheat as influenced by CR incorporation and P application under saline soil. 


\section{Effect of CR on soil physical status ECe, SAR and pH}

After the harvest of final DSR crop, overall 21\% decline in ECe with CR incorporation was observed as compared to before the sowing of DSR with CR incorporation (Table 6). Increasing the rate of $\mathrm{P}$ application under CR incorporation also caused a significant reduction in ECe being the maximum decline with higher rates of $\mathrm{P}_{2} \mathrm{O}_{5}$ (80 and $120 \mathrm{~kg} \mathrm{ha}^{-1}$ ) after completion of study. The ECe fell down to $3.79 \mathrm{dS} \mathrm{m}^{-1}$ against $4.15 \mathrm{dS} \mathrm{m}^{-1}$ before sowing of DSR due to CR incorporation and was exactly below the acceptable limit of soil ECe for successful crop growth and yield production. Similarly, under CR incorporation, there was also a considerable reduction in the value of SAR from 6.57 before crop sowing to 4.87 ( $\mathrm{mmol}_{\mathrm{c}}$ $\left.\mathrm{L}^{-1}\right)^{1 / 2}$ after the harvest of third season DSR crop. A minimum mean value of $\mathrm{pH}$ (8.15) was observed from the plots incorporated with CR. Decrease in $\mathrm{pH}$ value under $\mathrm{CR}$ incorporation along with $\mathrm{P}$ application was slightly higher as compared to control $\left(0 \mathrm{~kg} \mathrm{P}_{2} \mathrm{O}_{5}\right.$ ha $^{-1}$ ) plots.

Table 6: Physico-chemical analysis of saline soil before initiation and after the study during 2011-12 (Average of 3 repeats).

\begin{tabular}{|c|c|c|c|c|c|c|c|c|c|}
\hline & \multicolumn{3}{|c|}{ ECe } & \multicolumn{3}{|c|}{ SAR } & \multicolumn{3}{|c|}{ pH } \\
\hline & $+\mathbf{C R}$ & $-\mathbf{C R}$ & Mean & $+\mathbf{C R}$ & $-\mathbf{C R}$ & Mean & $+\mathbf{C R}$ & $-\mathbf{C R}$ & Mean \\
\hline & \multicolumn{9}{|c|}{ Before Sowing } \\
\hline 0 & $4.59^{\mathrm{NS}}$ & $4.58^{\mathrm{NS}}$ & $4.58^{\mathrm{NS}}$ & $6.57^{\mathrm{NS}}$ & 6.56 & $6.57^{\mathrm{NS}}$ & $8.38 \mathrm{NS}$ & $8.38^{\mathrm{NS}}$ & $8.38^{\mathrm{NS}}$ \\
\hline 40 & 4.57 & 4.57 & 4.58 & 6.56 & 6.57 & 6.57 & 8.39 & 8.37 & 8.38 \\
\hline 80 & 4.58 & 4.59 & 4.58 & 6.57 & 6.56 & 6.57 & 8.38 & 8.38 & 8.38 \\
\hline 120 & 4.58 & 4.58 & 4.58 & 6.56 & 6.57 & 6.57 & 8.38 & 8.39 & 8.38 \\
\hline \multirow[t]{2}{*}{ Mean } & $4.58 \mathrm{NS}$ & 4.58 & & $6.57 \mathrm{NS}$ & 6.57 & & $8.38 \mathrm{NS}$ & 8.38 & \\
\hline & \multicolumn{9}{|c|}{ After the Harvest of final Crop } \\
\hline 0 & $3.87 \mathrm{~d}$ & $4.58 \mathrm{a}$ & $4.22 \mathrm{~A}$ & $4.92 \mathrm{~d}$ & $6.57 \mathrm{a}$ & $5.74 \mathrm{~A}$ & $8.18 \mathrm{c}$ & $8.38 \mathrm{a}$ & $8.28 \mathrm{~A}$ \\
\hline 40 & $3.84 \mathrm{~d}$ & $4.51 \mathrm{~b}$ & $4.17 \mathrm{~B}$ & $4.87 \mathrm{de}$ & $6.45 \mathrm{~b}$ & $5.66 \mathrm{~B}$ & $8.16 \mathrm{c}$ & $8.37 \mathrm{a}$ & $8.27 \mathrm{~B}$ \\
\hline 80 & $3.69 \mathrm{f}$ & $4.42 \mathrm{c}$ & $4.06 \mathrm{C}$ & $4.82 \mathrm{e}$ & $6.42 \mathrm{bc}$ & $5.62 \mathrm{BC}$ & $8.11 \mathrm{e}$ & $8.34 \mathrm{~b}$ & $8.23 \mathrm{C}$ \\
\hline 120 & $3.77 \mathrm{e}$ & $4.38 \mathrm{c}$ & $4.08 \mathrm{C}$ & $4.85 \mathrm{de}$ & $6.36 \mathrm{c}$ & $5.61 \mathrm{C}$ & $8.14 \mathrm{~d}$ & $8.32 \mathrm{~b}$ & $8.23 \mathrm{C}$ \\
\hline Mean & $3.79 \mathrm{~B}$ & $4.47 \mathrm{~A}$ & & $4.87 \mathrm{~B}$ & $6.45 \mathrm{~A}$ & & 8.15 B & $8.35 \mathrm{~A}$ & \\
\hline
\end{tabular}

Means followed by same letter(s) do not differ significantly at $\mathrm{P} \leq 0.05 \mathrm{NS}=$ Non-significant.

\section{Soil Organic Matter (SOM) and P, K and Ca contents}

The data in Table 7 shows that at the end of study, there was a significant improvement in organic matter content as well as $\mathrm{P}$,
$\mathrm{K}$ and $\mathrm{Ca}$ availability owing to continuous $\mathrm{CR}$ incorporation and $\mathrm{P}$ fertilization. Maximum SOM, P, K and Ca contents were recorded with increased $\mathrm{P}$ application rate under CR incorporation in saline soil.

Table 7: Organic Matter $(\mathrm{OM})$ and Nutrient Status of saline soil before initiation and after the study during 2011-12 (Average of 3 repeats)

\begin{tabular}{|c|c|c|c|c|c|c|c|c|c|}
\hline & \multicolumn{3}{|c|}{ OM } & \multicolumn{3}{|c|}{$\mathbf{K}$} & \multicolumn{3}{|c|}{$\mathrm{Ca}$} \\
\hline & $+\mathrm{CR}$ & $-\mathbf{C R}$ & Mean & $+\mathrm{CR}$ & $-\mathbf{C R}$ & Mean & $+\mathrm{CR}$ & $-\mathbf{C R}$ & Mean \\
\hline & \multicolumn{9}{|c|}{ Before Sowing } \\
\hline 0 & $0.52^{\mathrm{NS}}$ & $0.54^{\mathrm{NS}}$ & $0.54^{\mathrm{NS}}$ & $78.03^{\mathrm{NS}}$ & $77.05^{\mathrm{NS}}$ & $77.02^{\mathrm{NS}}$ & $42.11^{\mathrm{NS}}$ & $43.04^{\mathrm{NS}}$ & $42.29^{\mathrm{NS}}$ \\
\hline 40 & 0.54 & 0.52 & 0.54 & 77.1 & 76.49 & 77.02 & 43.24 & 41.22 & 42.29 \\
\hline 80 & 0.53 & 0.54 & 0.54 & 79.09 & 77.02 & 77.02 & 42.73 & 41.57 & 42.29 \\
\hline 120 & 0.54 & 0.53 & 0.54 & 76.62 & 77 & 77.02 & 41.58 & 43.86 & 42.29 \\
\hline \multirow[t]{2}{*}{ Mean } & 0.54 & 0.54 & & $77.02 \mathrm{NS}$ & 77.02 & & $42.29 \mathrm{NS}$ & 42.29 & \\
\hline & \multicolumn{9}{|c|}{ After the Harvest of final Crop } \\
\hline 0 & $0.68^{\mathrm{NS}}$ & 0.49 NS & $0.59^{\mathrm{NS}}$ & $95.26 \mathrm{a}$ & $78.86 \mathrm{~d}$ & $87.06 \mathrm{~A}$ & $49.20 \mathrm{a}$ & $33.79 c$ & $41.50 \mathrm{~A}$ \\
\hline 40 & 0.69 & 0.52 & 0.61 & $93.46 \mathrm{~b}$ & $75.73 \mathrm{e}$ & $84.60 \mathrm{~B}$ & $48.55 \mathrm{ab}$ & $32.79 \mathrm{~d}$ & $40.67 \mathrm{~B}$ \\
\hline 80 & 0.71 & 0.53 & 0.62 & $88.48 \mathrm{c}$ & $73.86 \mathrm{e}$ & $81.17 \mathrm{C}$ & $48.13 \mathrm{~b}$ & $32.67 \mathrm{~d}$ & $40.40 \mathrm{~B}$ \\
\hline 120 & 0.7 & 0.54 & 0.62 & $91.75 \mathrm{~b}$ & $70.82 \mathrm{f}$ & $81.29 \mathrm{C}$ & $48.33 \mathrm{~b}$ & $32.76 \mathrm{~d}$ & $40.55 \mathrm{~B}$ \\
\hline Mean & $0.69 \mathrm{~A}$ & $0.52 \mathrm{~B}$ & & $92.24 \mathrm{~A}$ & $74.82 \mathrm{~B}$ & & $48.55 \mathrm{~A}$ & $33.00 \mathrm{~B}$ & \\
\hline
\end{tabular}

Means followed by same letter(s) do not differ significantly at $\mathrm{P} \leq 0.05 \mathrm{NS}=$ Non-significant. 


\section{Discussion}

\section{Growth and yield of DSR and Wheat}

Crop residue incorporation positively contributed in growth and yield of DSR particularly during second year. This was most probably due to complete decomposition and mineralization of added CR that enriched the soil with mineral nutrients in addition to improvement in soil physical condition by ameliorating toxic effects of hazardous ions. Moreover, water and $\mathrm{P}$ retention capacity might have also been improved due to added CR that retained comparatively excess moisture and $\mathrm{P}$ availability for a longer time. Besides, fabrication of acid farming substances by microbial activities and partial pressure of $\mathrm{CO}_{2}$ released during CR decomposition decreased soil $\mathrm{pH}$ and enhanced $\mathrm{P}$ availability and other necessary plant nutrients which encouraged healthy plant growth and hence yields. Crop residue incorporation upon decomposition substantially altered the nutrient stability in soils and reduced the adverse effect of saline ions. Similar points of view have also been documented by [11,25-27]. Further, adequate $\mathrm{P}$ fertilization promoted vigorous early plant growth, improved tillering and increased number of grains per panicle which eventually produced better yield of DSR $[12,28]$.

The increase in yield due to CR incorporation as well as P application have also been well documented by Eagle et al. [19], Slaton et al. [29], Sharma and Prasad [30], Krishna et al. [20], Aslam et al. [10], Ali et al. [31]. A significant increase in growth and yield components, straw and grain yields of wheat grown after DSR was observed with P fertilization and CR incorporation. This was definitely attributable to CR incorporation that reduced the intake of saline ions (Table 5) and enhanced PUE (Figure I). Besides, adequate $\mathrm{P}$ application and its judicious utilization further facilitated to improve growth and yield contributing parameters. As it have been discussed earlier, that CR incorporation upon decomposition substantially changed the nutrient balance in rhizosphere and microorganisms form symbiotic associations with plant roots increase the surface area and their access to P. Some microorganisms discharge acids into the soil which can help to solubilize little $\mathrm{P}$ minerals. Conversely, minimum number of productive tillers, panicle and spike lengths, DSR grains panicle ${ }^{-1}$, wheat grains spike-1 and yields in case of control $\left(0 \mathrm{~kg} \mathrm{P}_{2} \mathrm{O}_{5}\right.$ ha $\left.^{-1}\right)$ of both DSR and wheat crops were due to the P deficiency that directly distorted the normal tillering and grain formation by inhibiting their materialization and hence reduced yields $[32,33]$. When $\mathrm{P}$ was applied without CR incorporation, higher grain yield was obtained with increasing $\mathrm{P}$ rate and was the maximum where $\mathrm{P}$ was applied @120kg $\mathrm{P}_{2} \mathrm{O}_{5} \mathrm{ha}^{-1}$. Better crop growth and yield with maximum $\mathrm{P}$ application (120 $\mathrm{kg} \mathrm{P}_{2} \mathrm{O}_{5} \mathrm{ha}^{-1}$ ) under no CR incorporation could be due the reason that crops grown under salt-affected soils demands relatively higher nutrition to reach the potential yields $[8,10]$. But again this yield (harvested with $120 \mathrm{~kg}_{2} \mathrm{O}_{5}$ ha $^{-1}$ ) under no CR incorporation was, however, $6 \%$ less than obtained from $80 \mathrm{~kg} \mathrm{P}_{2} \mathrm{O}_{5}$ $\mathrm{ha}^{-1}$ with CR incorporation. It is obvious from the data in Table 3 that during second year of crop grown under CR incorporation, even lower $\mathrm{P}$ rate $\left(40 \mathrm{~kg} \mathrm{P}_{2} \mathrm{O}_{5} \mathrm{ha}^{-1}\right)$ performed moderately as equal to higher rate (120kg $\mathrm{P}_{2} \mathrm{O}_{5}$ ha $^{-1}$ with no CR) for both the crops. This happened most probably due to native P release from fixed sites due to acidified rhizosphere as a result of microbial activities during CR decomposition that enhanced its availability and ultimately grain yields. Moreover, fully decomposed CR and mineralization during subsequent wheat and DSR growing season presumably enriched the soil with mineral nutrients that contributed to a large extent in producing the maximum grain yields. Further, the longer root system in wheat crop might have absorbed nutrients from deeper soil layers to ensure healthy crop growth and hence yield. Thus, a higher application rate led to more P availability by enriching $\mathrm{P}$ content in the deeper layers where longer roots absorbed that residual $\mathrm{P}$, consequently allowing more $\mathrm{P}$ uptake to produce maximum yield.

There are several reports that signify the role of $\mathrm{P}$ application in the enhancement of crop yields [27,32,34]. Advantages of crop residue incorporation in soil and integrated nutrient management has been widely discussed by Byous et al. [17], Shiva et al. [35] and Ali et al. [31] who reported that a major part of nutrients (NPK) taken up by rice and wheat crops remains in the straw and become available for following crop plants upon decomposition. Similar conclusions have also been reported by Abid et al. [36], Sharma \& Prasad [30], Kharub et al. [37] and Yadvinder et al. [38]. Consequently, a substantial amount of $\mathrm{P}$ requirement for crop growth could be met by the incorporation of CR. Adequate $\mathrm{P}$ application approach on problem soils within the rice-wheat cropping system have to guarantee high and sustainable food grain production, high net profit and build-up of native soil P in available form.

\section{Ionic concentration in plant tissues, PUE and soil physical status}

The higher concentrations of $\mathrm{P}, \mathrm{K}^{+}$and $\mathrm{Ca}^{2+}$ in $\mathrm{CR}$ treated plots could be due to enhanced mineral nutrition in soil through complete decomposition and mineralization of incorporated CR, which improved the nutrient status in the plough layer and $\mathrm{P}$ application further facilitated their intake by crop plants because of synergistic effect with saline ions $[11,29]$. Phosphorus application particularly with CR incorporation considerably decreased $\mathrm{Na}^{+}$contents and increased $\mathrm{K}^{+}$and $\mathrm{Ca}^{2+}$ concentrations in plant tissues. Ali et al. [39] have reported that in the presence of relatively higher nutrients concentration in rhizosphere, plants absorbed and translocated relatively more $\mathrm{K}^{+}$and less $\mathrm{Na}^{+}$than at lower concentrations. The data indicated that $\mathrm{P}$ application still at lower rate $\left(40 \mathrm{~kg} \mathrm{P}_{2} \mathrm{O}_{5} \mathrm{ha}^{-1}\right)$ along with CR incorporation performed better or even statistically equal to higher dose of $\mathrm{P}$ without CR incorporation. These findings could also be supported by the results of Kinraide [40,41], Haq et al. [42], Delgado et al. [32] and Mahmood et al. [43] who reported that the root medium salinity interferes with the absorption and translocation of $\mathrm{K}^{+}$and $\mathrm{Ca}^{2+}$ by plants.

In the earlier discussion, it has been reported that enhanced $\mathrm{CO}_{2}$ partial pressure in the rhizosphere during CR decomposition process and microbial respiration increased the availability of released and added $\mathrm{P}$ as well as other essential nutrients. Crop residues incorporation upon decaying certainly modified the environment by adding organic matter and nutrients as well. 
This could be supported by the data in Tables 6 \& 7. Improved soil physical conditions further contributed to more $\mathrm{P}$ uptake and ultimately increased crop growth and yields. Under CR incorporation, additional increase in $\mathrm{P}$ application $\left(120 \mathrm{~kg} \mathrm{P}_{2} \mathrm{O}_{5}\right.$ ha 1) did not showed significant difference in PUE by both the crops. On the other hand, when $\mathrm{P}$ was applied without CR incorporation, maximum PUE was determined from the treatments where $\mathrm{P}$ was applied at higher rate $\left(120 \mathrm{~kg} \mathrm{P}_{2} \mathrm{O}_{5} \mathrm{ha}^{-1}\right)$ and was not as much of $\mathrm{P}$ application @ 80 $\mathrm{kg} \mathrm{P}_{2} \mathrm{O}_{5} \mathrm{ha}^{-1}$ under CR incorporation. This was most probably due to excessive uptake of saline ions in the form of $\mathrm{Na}_{2} \mathrm{PO}_{4}$ compounds [11] that comparatively reduced biomass production of both the crops which resulted in relatively less PUE. Further the retention of $\mathrm{P}$ in soil in the forms that are not directly available to plants is, fundamentally, an intrinsic soil characteristic. Many studies have investigated the effects of $\mathrm{pH}$ variation on $\mathrm{P}$ retention and availability, but reliable enhancement in soil P availability has not yet been obtained. However, minor changes may be achievable. For example, evidences from many detailed studies show that the more organic matter there is in soil, the more the amount of very readily-available plant $\mathrm{P}[31,44-46]$. Our results indicated that $\mathrm{P}$ application @ 80 kg $\mathrm{P}_{2} \mathrm{O}_{5} \mathrm{ha}^{-1}$ along with CR incorporation (2t ha $\left.{ }^{1}\right)$ was found to be superior than rest of the treatments in terms of producing maximum grain yields of both DSR and wheat crops grown under marginally saline soil $[47,48]$. During second year of DSR grown under CR incorporation, the lower $\mathrm{P}$ application rate $(40 \mathrm{~kg}$ $\mathrm{P}_{2} \mathrm{O}_{5}$ ha $^{-1}$ ) performed slightly better and produced comparable paddy yield as that of higher $\mathrm{P}$ application rate $\left(120 \mathrm{~kg} \mathrm{P}_{2} \mathrm{O}_{5}\right.$ ha-1 $\left.^{-1}\right)$ without CR incorporation. Therefore, continuous CR incorporation is worth recommended to restore soil fertility as well as productivity.

\section{References}

1. Qureshi RH, Qadir M (1992) Is the reclamation of dense saline sodic soils uneconomical? Myths and Facts. Pak J Agric Sci 29: 317-18.

2. Ahmad N (2002) Soil fertility management: Key to food security and poverty alleviation. Proc $12^{\text {th }}$ Natl Cong Soil Sci NIAB Faisalabad, Pakistan, pp. 17-18.

3. Ahmad N, Qureshi RH, Qadir M (1990) Irrigation systems management/ research (Biotic and chemical reclamation of sodic soil). $4^{\text {th }}$ Ann Report pp. 1989-1890.

4. Ghafoor A, Shahid MI, Saghir M, Murtaza G (1992) Use of high Mg brackish water on phosphogypsum and FYM treated saline-sodic soil. I. Soil improvement. Pak J Agric Sci 29: 20-24.

5. Minhas PS (1996) Saline water management for irrigation in India. Agric Water Management 30(1): 1-24.

6. Qadir M, Steffens D, Schubert E (2003) Sodium removal from a calcareous saline-sodic soil through leaching and plant uptake during phytoremediation. Land Degr Dev 14(3): 301-307.

7. Maser P, Gierth M, Schroeder JI (2002) Molecular mechanisms of potassium and sodium uptake in plants. Plant \& Soil 247(1): 43-54.

8. Mahmood IA, Aslam M, Qureshi RH, Nawaz S (1994) Phosphorus nutrition of rice in a salt-affected soil fertilized with various nitrogen sources. Pak J Agri Sci 31: 168-173.

9. Ghafoor A, Qadir M, Murtaza G (2004) Salt-affected soils: Principles of Management. Allied Book Centre, Urdu Bazar, Lahore, Pakistan.

10. Aslam M, Flowers TJ, Qureshi RH, Yeo AR (2008) Interaction of phosphate and salinity on the growth and yield of rice (Oryza sativa L.). J Agron Crop Sci 176(4): 249-258.
11. Mahmood IA, Ali A, Aslam M, Shahzad A, Sultan T, et al. (2013) Phosphorus availability in different salt-affected soils as influenced by crop residue incorporation. Intl J Agric Biol 15: 472-478.

12. Sainio PP, Kontturi M, Peltonen J (2006) Phosphorus seed coating enhancement on early growth and yield components in Oat. Agron J 98(1): 206-211.

13. NFDC (2012) Fertilizer use related statistics. National Fertilizer Development Centre, Planning Division, Government of Pakistan, Islamabad, Pakistan.

14. Qayyum MA, Malik MD (1988) Farm production in salt-affected soils. In: Managing Soil Resources. ${ }^{\text {st }}$ National Congress of Soil Science, Lahore, Pakistan, pp. 356-364.

15. Rahmatullah, Gill MA, Shaikh BZ, Salim M (1994) Bioavailability and distribution of $\mathrm{P}$ among inorganic fractions in calcareous soils. Arid Soil Res Rehab 8(3): 227-234.

16. Saleem M, Ahmad TN, David JG (1986) Fertilizers and their use in Pakistan. Planning and Development Division, Govt. of Pakistan, National Fertilizer Development Center, Islamabad, Pakistan.

17. Byous EW, Williuams JE, Jonesa GE, Horwath WR, Kessel C (2004) Nutrient requirements of rice with alternative straw management. Better Crops 88(3): 6-11.

18. Misra RD, Gupta VK, Pandey DS (1996) Management crop residue in rice. Intal Rice Res Notes 21: 71-72.

19. Eagle AJ, Bird JA, Horwaath WR, Linquist BA, Brouder SM, et al. (2000) Rice yield and nitrogen utilization efficiency under alternative straw management practices. Agron J 92(6): 1096-1103.

20. Krishna GMA, Misra AKK, Hati KM, Bandyopadhyay KK, Ghosh PK, et al. (2004) Rice residue management options and effects on soil properties and crop productivity. Food, Agri \& Environ 2: 224-231.

21. Ryan J, Estefan G, Rashid A (2001) Soil and plant analysis laboratory manual, International Center for Agricultural Research in the Dry Areas (ICARDA), Aleppo, Syria, p. 172.

22. Rhoades JD (1982) Cation Exchange Capacity. In: Methods of Soil Analysis. Part 2. Chemical and microbiological properties. AL Page (ed.). Amer Soc Agron Madison, Wisconsin, USA, pp. 149-158.

23. Fageria NK, Baliger VC, Jones CA (1997) Growth and mineral nutrition of field crops. $2^{\text {nd }}$ Edn Marcel Dekker Inc New York, USA.

24. Gomez KA, Gomez AA (1984) Statistical procedure for agricultural research, $2^{\text {nd }}$ edn., John Wiley and Sons, New York, USA.

25. Rath AK, Ramakrishnan B, Rao VR, Sethunathan N (2005) Effects of ricestraw and phosphorus application on production and emission of methane from tropical rice soil. J Plant Nutr \& Soil Sci 168(2): 248-254.

26. Mishra BN, Kumar D, Shivay YS (2006) Effect of organic sources on productivity, grain quality and soil health of rice (Oryza sativa)-wheat (Triticum aestivum) cropping system. In: Ahlawat et al. (Eds.), Extended Summaries of Golden Jubilee National Symposium on Conservation Agriculture and Environment, Varanasi, India, p. 280.

27. Danga BO, Wakindiki IIC (2009) Effect of placement of straw mulch on soil conservation, nutrient accumulation, and wheat yield in a humid Kenyan highland. J Trop Agric 47(1-2): 30-36.

28. Arshadullah M, Ali A, Hyder SI, Khan AM (2012) Effect of wheat residue incorporation along with $\mathrm{N}$ starter dose on rice yield and soil health under saline sodic soil. J Anal \& Plant Sci 22: 753-757.

29. Slaton NA, Wilson CE, Norman RJ, Ntamatungiro S, Frizzell DL (2002) Rice response to phosphorus fertilizer application rate and timing on alkaline soils in Arkansas. Agron J 94(6): 1393-1399.

30. Sharma SN, Prasad R (2003) Yield and P uptake by rice and wheat grain in a sequence as influenced by phosphate fertilization with diammonium phosphate and Mussoorie rock phosphate with or without crop residue and phosphate solubilizing bacteria. J Agric Sci 141(3-4): 359-369. 
31. Ali A, Arshadullah M, Hyder SI, Mahmood IA, Zaman B (2012) Rice productivity and soil health as affected by wheat residue incorporation along with nitrogen starter dose under salt-affected soil. Pak J Agric Res 25: 257-265.

32. Delgado A, Uceda I, Andreu L, Kassem S (2002) Fertilizer phosphorus recovery from gypsum-amended reclaimed calcareous marsh soils. Arid land Res Manage 16(4): 319-334.

33. Rehim A, Ranjha AM, Rahamtullah, Waraich EA (2010) Effect of phosphorus application and irrigation scheduling on wheat yield and phosphorus use efficiency. Soil Environ 29(1): 15-22.

34. Van der Eijk D, Janssen BH, Oenema O (2006) Initial and residual effects of fertilizer phosphorus on soil phosphorus and maize yields on phosphorus fixing soils. A case study in Southwest Kenya. Agric Ecol Managmt 116(1-2): 104-120.

35. Shiva D, Vyas AK, Shivay YS, Kumar D, Kumar A (2012) Yield of whea and residual soil fertility under organic and inorganic fertilization. In: Proc Natl Seminar on Indian Agriculture: Preparedness for Climate Change, March 24-25, 2012, New Delhi (Shiva D, Barthakur S, Prajapati K, Annapurna K, Satyavathi T, et al. (Eds.), Organized by Indian Society of Agricultural Science, Div. Agron. IARI New Delhi, India, pp. 139-140.

36. Abid M, Ahmad F, Ahmad N, Ahmad I (2002) Effect of phosphorus on growth, yield and mineral composition of wheat in different textured saline sodic soils. Asian J Plant Sci 1(4): 472-475.

37. Kharub AS, Sharma RK, Mongia AD, Chhokar RS, Tripathi SC, et al. (2004) Effect of rice (Oryza sativa) straw removal, burning and incorporation on soil properties and crop productivity under rice-wheat (Triticum aestivum) system. Ind J Agric Sci 74(6): 295-299.

38. Yadvinder S, Bijay S, Ladha JK, Khind CS, Khera TS, et al. (2004) Effects of residue decomposition on productivity and soil fertility in rice-wheat rotation. Soil Sci Soc Amer J 68(3): 854-864.

39. Ali A, Salim M, Ahmad I, Mahmood IA, Zaman B, et al. (2003) Nutritional role of calcium on the growth of Brassica napus under saline conditions. Pak J Agric Sci 40(3-4): 106-113.
40. Kinraide TB (1998) Three mechanisms for the calcium alleviations of mineral toxicities. Plant Physiol 118: 513-520.

41. Kinraide TB (1999) Interactions among $\mathrm{Ca}^{+2}, \mathrm{Na}^{+}$and $\mathrm{K}^{+}$in salinity toxicity: Quantitative resolution of multiple toxic and ameliorative effects. J Exp Bot 50(338): 1495-1505.

42. Haq I, Khattak SG, Rehman H, Ali A, Salim M (2001) Effect of various amendments on the yield of rice crop under saline-sodic conditions in Mardan/Swabi District. Intl J Agric Biol 3: 289-291.

43. Mahmood IA, Ali A, Salim M, Arshadullah M, Zaman B, et al. (2009) Impact of calcium sulphate and calcium carbide on nitrogen use efficiency of wheat in normal and saline sodic soils. Soil \& Environ 28: 29-37.

44. Manske GGB, Ortiz Monasterial JI, M van Ginkel R, Gonzalez RM, Fischer RA, et al. (2000) Importance of P-uptake efficiency vs. Putilization for wheat yield in acid and calcareous soils in Mexico. European J Agron 14(4): 261-274.

45. Leytem AB, Mikkelsen RL (2005) The nature of phosphorus in calcareous soils. Better Crops 89(2): 11-13.

46. Rehim A, Hussain M, Abid M, Zia-ul-Haq M, Ahmad S (2012) Phosphorus use efficiency of Trititicum aestivum L. as affected by band placement of phosphorus and farmyard manure on calcareous soils. Pak J Bot 44(4): 1391-1398.

47. Mishra BN, Kumar D, Singh N (2005) Effect of organic sources of plant nutrition on productivity and soil health of rice-wheat cropping system. In: Mishra BN, Kumar D, Singh N (Eds.), National Symposium on Agrophysical Approaches in Disaster Mitigation, Resource Management and Environmental Protection. Organized by Indian Society of Agrophysics, Division of Agricultural Physics, IARI, New Delhi, India, pp. 17-19.

48. NFDC (2010) Fertilizer use related statistics. National Fertilizer Development Centre, Planning Division, Government of Pakistan, Islamabad, Pakistan. 